\title{
L'appréhension de la notion de risques et ses différentes déclinaisons en géographie
}

\section{Understanding the notion of risk and its various forms in geography}

\author{
Anthony Tchékémian ${ }^{1}$ \\ ${ }^{1}$ Maître de conférences Géographie et Aménagement du territoire, Université de la Polynésie Française, UMR 241 EIO \\ anthony.tchekemian@upf.pf
}

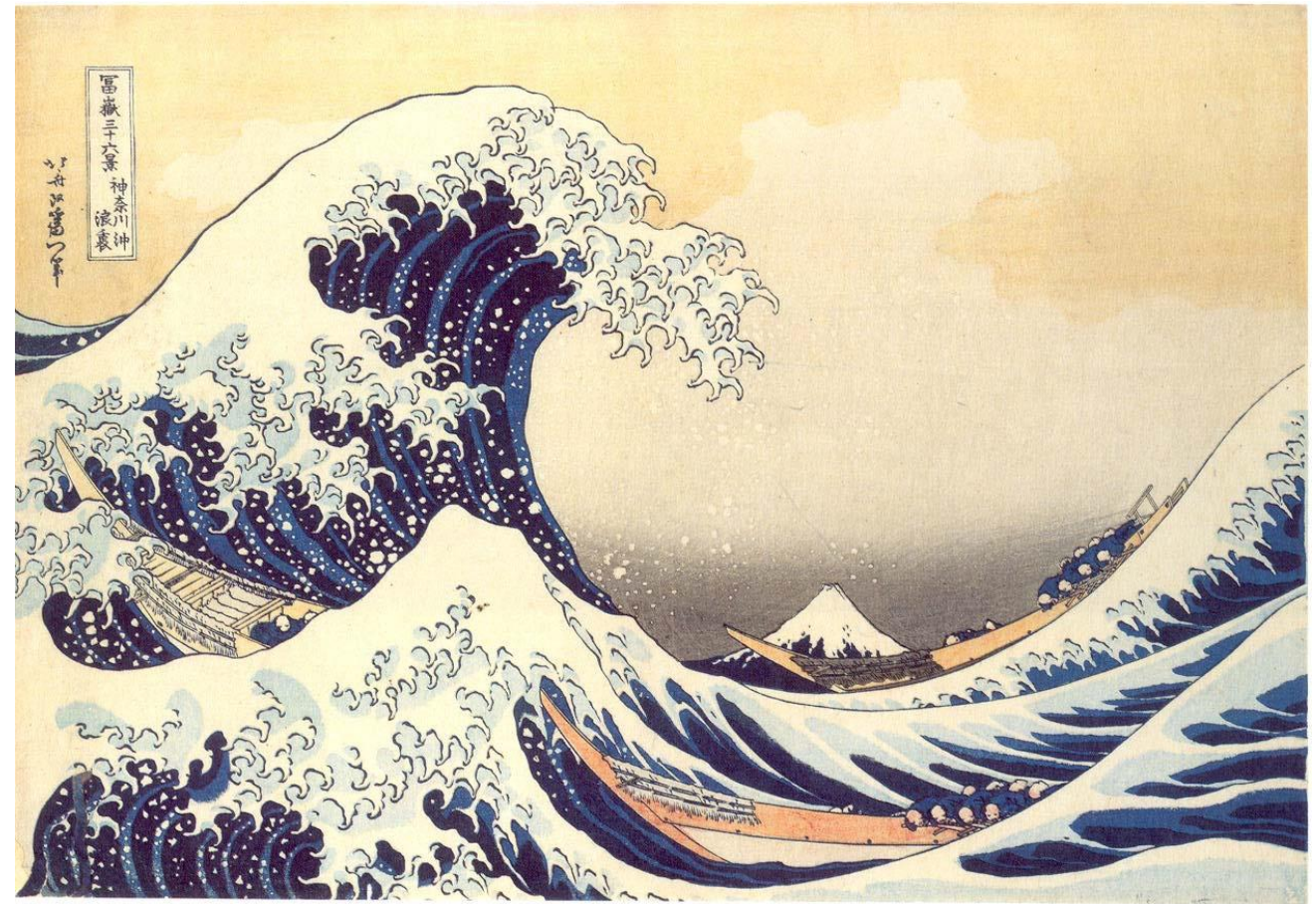

Figure 1. Hokusai, La Grande Vague de Kanagawa, estampe publiée en 1830-1831 (gravure sur bois nishiki-e, $25 \mathrm{~cm} \times 37 \mathrm{~cm}$, ōban yoko-e, Metropolitan Museum of Art, New York).

RÉSUMÉ. A partir de la première industrialisation, en Europe, en Amérique, au Japon, les explorations, l'usage du charbon, de l'acier, du nucléaire ou des nanotechnologies ont permis d'améliorer le niveau de vie des populations, même si cette forme de développement est de plus en plus remise en cause. Toutefois, ces avancées auraient-elles été possibles si leurs acteurs avaient d'emblée eu conscience de leurs conséquences humaines et environnementales ?

Désormais, les sociétés revendiquant des valeurs écologiques tentent, pourtant, de limiter les conséquences du risque par la réglementation, voire l'interdiction de l'action dangereuse, illustrée par le «principe de précaution ». Dans ce contexte, l'article interroge la place de la géographie dans la réflexion sur le risque et son acceptation. II plaide pour une approche transdisciplinaire, intégrant les perceptions et les représentations du risque à l'étude des territoires.

ABSTRACT. From the early stages of industrialization in Europe, America or Japan, the living standards of populations have drastically improved thanks to explorations, the use of coal, steel, as well as of nuclear or nanotechnologies, although such progress is increasingly called into question. However, would these improvements have been possible had their initiators been aware of the human and environmental consequences right from the beginning?

Nowadays, companies boasting ecological values attempt to limit the consequences of risks by regulating or even prohibiting any action deemed dangerous, as illustrated by the "precautionary principle". In this context, this paper offers a reflection on the importance given to geography when it comes to dealing with the notion of risk and accepting it, arguing for a transdisciplinary approach, thus integrating perceptions and representations of the risk concept into the study of territories.

MOTS-CLÉS. risques, développement, société, acceptation, Occident, géographie.

KEYWORDS. risks, development, society, acceptance, West, geography. 
«Conscients des périls et des souffrances qui pèsent sur l'humanité et sur la planète, nous ne voudrions pas nous laisser aller à la peur et à la résignation. Pourtant le bel espoir humain est sans cesse menacé par le désenchantement. Les difficultés économiques de plus en plus lourdes, la complexité parfois écrasante des sociétés, l'impuissance face aux catastrophes naturelles tendent à étouffer les pousses d'espérance. ». (Alois, 2012)

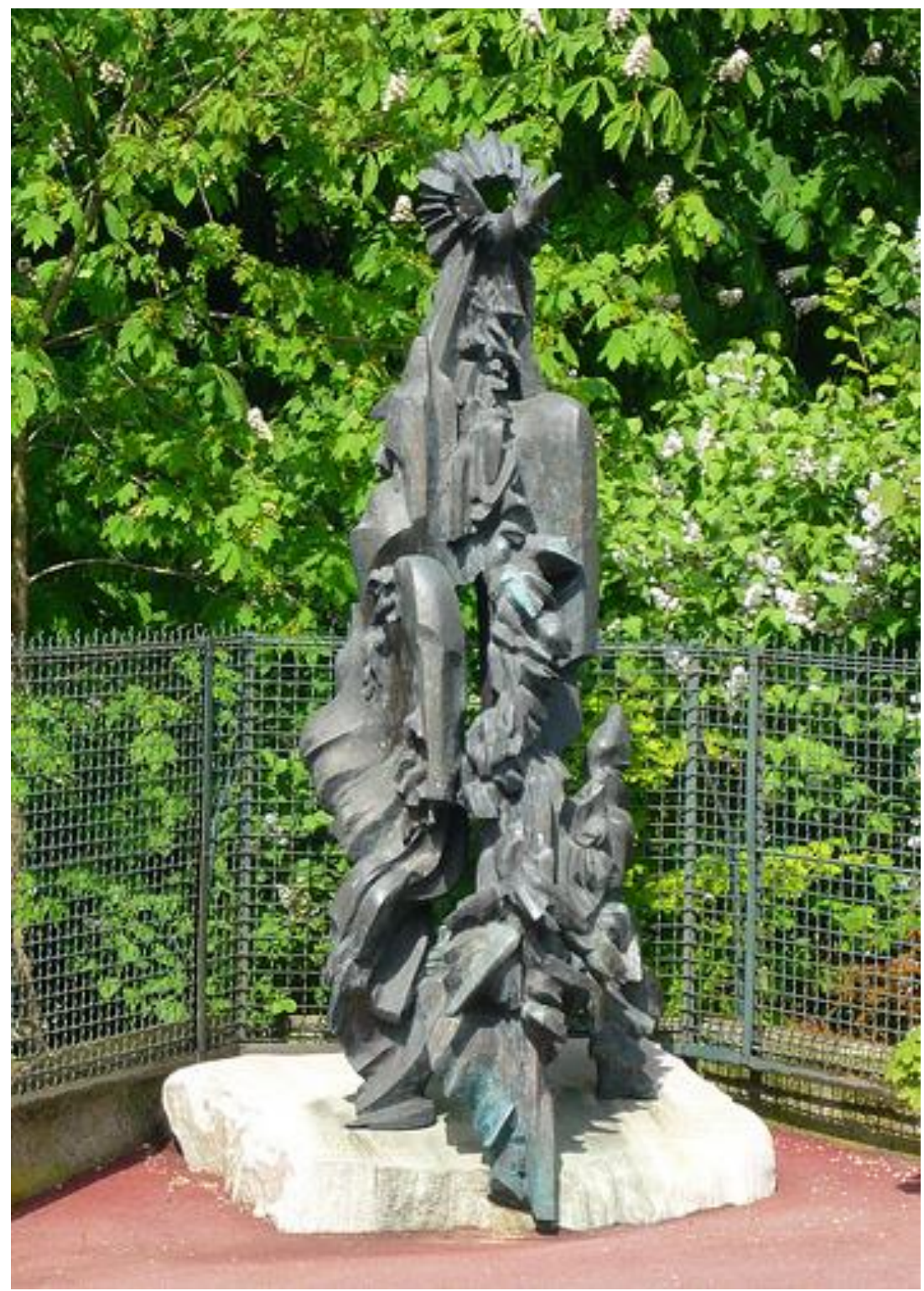

Figure 2. Sculpture d'Albert Avetisyan, Les Oiseaux (1992), offerte à la commune d'Issy-les-Moulineaux, en remerciement de l'aide humanitaire apportée à l'Arménie suite au séisme de 1988.

Source : Ville-issy.fr

\section{Introduction}

De façon générale, le risque se définit comme un danger plus ou moins prévisible, c'est-à-dire «la probabilité qu'un évènement ait des conséquences généralement considérées comme dommageables. Puis, si le risque désigne « ce qui peut advenir et que l'on ne voudrait pas avoir à subir directement ou indirectement (par ses conséquences), on caractérise une catégorie du rapport au monde qui, tout en présentant un caractère très général, peut prendre des formes différentes et faire l'objet de constructions diverse » (Bourdin, 2003 : 13). Le risque doit être évalué dans tout processus de prise de décision. Le sociologue Ulrich Beck (2001) a fait de cette notion une caractéristique des sociétés contemporaines »(Dollo, 2017: 325). Ainsi, dans cette étude, la notion de risque constitue le fil conducteur permettant aussi bien d'interroger l'action publique - en matière de gestion - que les 
connaissances et les représentations - sur le plan des perceptions des risques - ou encore les comportements individuels - en ce qui concerne la prise de risque.

En géographie, la notion de « risque $»^{2}$ se définit comme « la possibilité ou la probabilité d'atteinte aux enjeux humains (personnes, biens, valeurs culturelles...)»(D'Ercole, Pigeon, 1999 : 340). Le risque peut s'appliquer à une personne, à une population, à des biens matériels, à un environnement ou encore à un milieu naturel. Qu'ils s'agissent de risques naturels, industriels, technologiques, sociaux, mineurs ou majeurs ${ }^{3}$, ils constituent une notion importante dans le champ disciplinaire de la géographie, notamment dans les domaines de l'aménagement du territoire, de l'urbanisme, de l'environnement, de l'industrie), mais aussi de la finance, du droit, de la santé et des assurances. Par exemple, à la fin des années quatre-vingt, Georges-Yves Kervern, polytechnicien, ingénieur des mines et diplômé de l'Institut d'études politiques de Paris, conçoit un néologisme par la science des « cindyniques », du grec ancien kíndunos (danger), étudie les dangers, les risques. L'approche associe des sciences (météorologie et géologie), des catastrophes (chimies, physiques ou naturelles) et des aspects humains (économie, urbanisme, psychologie). Cette science s'intéresse principalement aux risques industriels et naturels (Kervern, Rubise, 1991 ; Kervern, 1999 ; Kervern, Boulanger, 2007). Puis, en 2004, dans Le risque, cet inconnu, Georges Jousse (2009) propose le terme « riscologie » en vue de l'étude générale et scientifique des risques, quels qu'ils soient.

Cependant, l'étude de la conscience du risque et de la prise de risque demeure marginale en géographie, même si les géographes ont très tôt analysé la gestion du risque et les interactions entre nature et société. Par exemple, Roger Dion, dans son ouvrage Le Val de Loire : étude de géographie régionale, s'est intéressé, dès 1934, à la gestion des territoires au travers des inondations et de la protection des populations contre celles-ci, sans toutefois employer le mot «risque» (VeyretMekdjian, 2001 : 20-21). Or, « l'idée de risque découle de la prise de conscience et de la dénonciation de la dégradation des ressources naturelles par les sociétés, et cela dès le XVIIIe siècle » (Veyret, Reghezza, 2005b : 3).

Ainsi, la conscience est la perception qu'un être humain a de lui-même, de sa propre existence, ainsi que du monde extérieur. Cependant, ce terme est souvent associé à d'autres qualificatifs (conscience sociale, collective, etc.). «La conscience sociale, ou conscience de la société, est inséparable de la conscience de soi-même, parce que nous pouvons à peine penser à nous-mêmes sans référer à un groupe social, quel qu'il soit, pas plus que le groupe ne peut se penser sans référence à nous-mêmes. [...] ce dont nous sommes vraiment conscients est un ensemble personnel ou social plus ou moins complexe, dont nous accentuons à un moment donné le caractère particulier, et à un autre, le caractère général. » (Cooley C. H., 2016). La conscience que les individus sociaux ont de leur action n'est pas science ; la conscience peut être comprise comme vraie dès lors qu'on la rattache à la façon dont un agent social calcule ses intérêts (Akoun, 2001: 45). «La conscience du risque résulte en général du déroulement d'une crise ou d'une catastrophe ; à partir de cet événement souvent dramatique et qui peut se reproduire, on réalise ce que l'on nomme "un retour d'expérience" qui conduit à la prise de conscience du risque. Le risque se décline en termes de prévention, il doit être intégré aux politiques d'aménagement du territoire tandis qu'il convient d'anticiper la catastrophe afin d'établir des scénarii adaptés permettant la gestion la plus rapide et la plus efficace de l'évènement lors de son déclenchement. » (Veyret, Chocat, 2005).

Quant à la notion de prise de risque, elle permet d'élargir et de transformer la problématique de la décision. Identifier le moment ou la nature de la décision est souvent chose difficile, qu'il s'agisse de processus collectifs ou de comportement individuel. Souvent l'action se construit de manière continue

\footnotetext{
${ }^{2}$ A ne pas confondre avec la « catastrophe » : le risque, fait référence à une possibilité, tandis que la catastrophe est un risque qui se concrétise. En principe, l'étude des catastrophes devrait éviter leur répétition.
}

${ }^{3}$ Le risque majeur se caractérise par une faible fréquence et de graves dommages sur les humains, les biens et l'environnement. 
que l'on peut certes réduire à de microdécisions, mais sans que cela soit vraiment éclairant. L'avantage du raisonnement sur les prises de risques est qu'il échappe à la recherche du moment de la décision, qu'il permet d'analyser des séquences de comportement (sans avoir à rechercher obstinément un point de départ), et qu'il introduit l'analyse des différences entre ce qui est perçu par les acteurs concernés et ce que l'on peut mesurer à partir d'une norme extérieure visant l'objectivité » (Bourdin, 2003).

Le risque resta longtemps le domaine réservé des géographes physiciens, qui s'intéressaient essentiellement à l'aléa. Il fallut attendre les années soixante-dix pour que la vulnérabilité soit intégrée à la discipline (Veyret, Reghezza, 2005 : 63 ; Becerra, 2012). Aujourd'hui encore, la prise en charge du risque par la géographie apparaît partielle, d'autant que les chorèmes, à savoir des schématisations visuelles de territoires, apparus dans les années quatre-vingt pour «résoudre en géographie la contradiction de fond entre général et particulier, loi et individu, nomothétie et idiographie... » (Brunet, 2006 : 97), sont désormais abandonnés. Pourtant, par son approche généraliste et globalisante, cette forme de modélisation aurait pu contribuer à élaborer une réflexion globale sur le risque. D'ailleurs, pour Laurini et Servigne (2011), les chorèmes sont «de potentiels systèmes de représentations visuelles de connaissances spatiales, [...] des outils de résumés visuels de bases de données géographiques et [des] systèmes d'accès à de telles bases. Les directions de recherches touchent aussi bien les aspects cognitifs, sémantiques, sémiologiques, fouille de données spatiales, interopérabilité, etc. ».

Désormais, l'étude des risques s'oriente essentiellement vers l'articulation de la prédiction à la prévention et vers la compréhension des risques. Par sa tentative d'établir des indicateurs, la géographie contemporaine apparaît alors comme une discipline-diagnostic déterminant des seuils d'acceptabilité des risques. La cartographie y joue un rôle essentiel, en lien avec d'autres disciplines : l'urbanisme, l'architecture, l'aménagement du territoire ou le droit, mais aussi la climatologie, la météorologie, la géologie, la physique, les mathématiques, l'informatique et les sciences de l'environnement. Il s'agit de mieux comprendre les rapports entre nature et société (Ferrier, 1998 ; Veyret, Reghezza, 2005), tout en y associant les réflexions d'autres sciences sociales, comme la sociologie, la philosophie, l'histoire, l'archéologie et l'anthropologie. C'est pourquoi les études géographiques sur les risques et leurs origines, vus de façon rétrospective, se multiplient (Leone, Vinet, 2011 : 17). La catastrophe ${ }^{7}$, la gestion du risque ${ }^{8}$ et la mémoire ${ }^{9}$ de celui-ci retiennent principalement l'attention des chercheurs.

Outre sa dimension spatiale, le risque doit être replacé dans une perspective diachronique. Si la notion de risque semble anachronique pour les sociétés anciennes, l'exploit y trouvait cependant sa place dans un idéal héroïque, il n'est que de penser à Achille ou Héraclès, sans parler des athlètes,

\footnotetext{
${ }^{7}$ Par exemple : Albouy F.-X., (2002), Le Temps des catastrophes. Descartes et Cie, 176 p. ; Clavandier G., (2004), La mort collective. Pour une sociologie des catastrophes. CNRS Éditions, 255 p. ; Fogwe N.Z. et Tchiadeu G., (2006), "On cindynoligy in Cameroon geography: From crises to catastrophes", in Mutibe, n², pp 115-125 ; Jeudy H.-P., (1990), Le désir de catastrophe. Ed. Aubier ; Girard V., Langumier J., (2006), "Risques et catastrophe. De l'enquête de terrain à la construction de l'objet ", Genèses, ${ }^{\circ} 63, \mathrm{pp}$ 128-142 ; Pagney P., (1994) Les catastrophes climatiques. PUF, collection Que sais-je ?, Paris, 128 p.

${ }^{8}$ Par exemple : Allard P., (2001), Éléments pour une problématique de l'histoire du risque. Du risque accepté au risque maîtrisé. Représentations et gestion du risque d'inondation en Camargue, XVIIle-XIXe siècles. Mémoire pour l'Habilitation à la direction de recherches, Revue d'histoire du XIXe siècle, n²3, pp. 289-294 ; Chaline C., Dubois-Maury J., (1994), La ville et ses dangers. Prévention et gestion des risques naturels, sociaux et technologiques. Masson, coll. Pratiques de la géographie, Paris, 247 p. ; D’Ercole R., Metzger P., (2005), « Repenser le concept de risque pour une gestion préventive du territoire », Pangea, juin-décembre, pp. 19-36 ; Lagadec P., (1991), "La Gestion des crises », Ediscience, Paris, pp. 54-57 ; Veyret Y., Meschinet de Richemond N., (2004), Géographie des risques naturels en France. De l'aléa à la gestion. Ed. Hatier, 251 p.

${ }^{9}$ Notamment en raison du recul général du structuralisme et de la préférence pour l'interprétation par l'historicisation. Par exemple : Mavoungo J., (2008), Le séisme majeur du 11 janvier 1839 de la Martinique. Elément de mémoire collective, d'éducation et de prévention du risque sismique aux Antilles Françaises. Ed. Publibook université, 226 p.
} 
comme Milon de Crotone ${ }^{11}$. Cet idéal de l'exploit était toutefois éloigné de notre notion de risque et s'intégrait à une représentation tragique du monde : «celui que les dieux aiment meurt jeune », écrivait le poète Ménandre (Létoublon, 2012: 282). Cependant, à côté de cet idéal héroïque émergea un nouveau rapport à l'investissement économique via le développement du grand commerce maritime dans le monde égéen aux $\mathrm{V}^{\mathrm{e}}-\mathrm{IV}^{\mathrm{e}}$ siècles av. J-C. Le «prêt à la grosse aventure » intégra, en effet, le risque à l'investissement économique ; «l'emprunteur n'est donc pas libre d'utiliser cet argent comme il l'entend, mais seulement de la manière dont le contrat le stipule »(Bresson, $2008: 68$ ). Par la suite, dans l'Italie de la Renaissance, armateurs et négociants s'associèrent pour transporter les marchandises, tandis que les premières compagnies d'assurance - au travers de l'expression "périls encourus en mer » - estimèrent et évaluèrent les risques inhérents à la navigation maritime. Dans ce contexte, le français «risque » dériverait de l'italien médiéval risco, et serait lié à l'idée de péril en mer. Son étymologie tiendrait au caractère tranchant des rochers sur lesquels s'échouaient les navires (Bloch, 1960). Puis, le mot «risque » apparaît en français au XVI ${ }^{\mathrm{e}}$ siècle, dans les calculs de probabilité des assureurs. Il qualifie les évènements aléatoires représentant un danger, une perte potentielle. $\mathrm{Au} \mathrm{XVII}{ }^{\mathrm{e}}$ siècle, Pascal et le Chevalier de Méré systématisèrent cet emploi du mot «risque » (Veyret et al., $2004: 19)$.

A l'origine économique, la prise en compte de la notion de risque s'étendit ensuite à la perception des catastrophes. En 1755, l'émotion suscitée par le tremblement de terre de Lisbonne, suivi d'un razde-marée et d'un incendie qui ravagèrent la quasi-totalité de la capitale portugaise ${ }^{14}$, «laïcise le danger, en même temps qu'elle accorde à la société une place dans la définition du risque ». Suite à cette catastrophe, des philosophes du Siècle des Lumières, tels que Kant, Rousseau, Diderot et Voltaire, s'opposèrent aux thèses optimistes de Pope, Wolf et Leibniz (1710), lequel désignait par le terme théodicée, «la justification de la bonté de Dieu, en dépit du mal inhérent au monde » (Duméry, 2017). Les autorités religieuses continuèrent de proclamer que le désastre était une manifestation de la colère divine. Toutefois, une violente polémique opposa Rousseau, pour qui construire une ville côtière dans une zone sismique relevait de la responsabilité exclusive des hommes, à Voltaire, qui accusait le hasard et le malheureux concours de circonstances. La position de ce dernier est présente dans Poème sur le désastre de Lisbonne (1756) et Candide (1759). L'Europe connait alors « un tournant fondamental dans l'appréhension du danger : pour Rousseau ${ }^{15}$, c'est l'homme (et non Dieu) qui est responsable, car il a construit la ville dans un secteur à risque » (Veyret-Mekdjian, 2001 : 22-23). La perception du risque passe de l'approche économique à l'analyse spatiale des catastrophes. Progressivement, le risque va se définir comme le produit d'un aléa et d'une vulnérabilité (Poirier, 2005).

\footnotetext{
${ }^{11}$ Voir Roubineau (2016) ; puis, sur l'importance de l'exploit militaire dans les processus de légitimation politique voir Bouyssou (2016 : 30-31).

${ }^{14}$ En ce prospère XVIII ${ }^{\mathrm{e}}$ siècle, Lisbonne est l'une des plus grandes villes d'Europe, avec près de 275000 habitants ; elle joue un rôle crucial dans le commerce mondial et elle est aussi un centre majeur de la religion catholique. Le $1^{\text {er }}$ novembre 1755 , un violent séisme de magnitude 8,5 à 9 sur l'échelle de Richter, suivi d'un tsunami (trois vagues successives de 5 à 15 mètres de haut), puis d'une série d'incendies détruisit la plus grande partie de Lisbonne, faisant plusieurs dizaines de milliers de victimes (Bouhdiba, 2014).
}

\footnotetext{
${ }^{15}$ Suite cette catastrophe, des philosophes du siècle des lumières, tels que Kant, Rousseau, Diderot et Voltaire s'opposèrent aux thèses optimiste de Pope, Wolf et de Leibniz, lequel par le terme « théodicée " (1710) désignait « la justification de la bonté de Dieu, en dépit du mal inhérent au monde " (selon Henry DUMÉRY, définition de " Théodicée », dans Encyclopædia Universalis, [URL : http://www.universalis-edu.com/encyclopedie/theodicee/], consulté le 11 septembre 2017. Les autorités religieuses continuèrent de proclamer que le désastre était une manifestation de la colère divine. Toutefois, une violente polémique opposa Rousseau, pour qui construire une ville côtière dans une zone sismique relevait de la responsabilité exclusive des hommes, à Voltaire, qui accusait le hasard et le malheureux concours de circonstance. La position de ce dernier est présente dans Poème sur le désastre de Lisbonne (1756) et Candide (1759).
} 


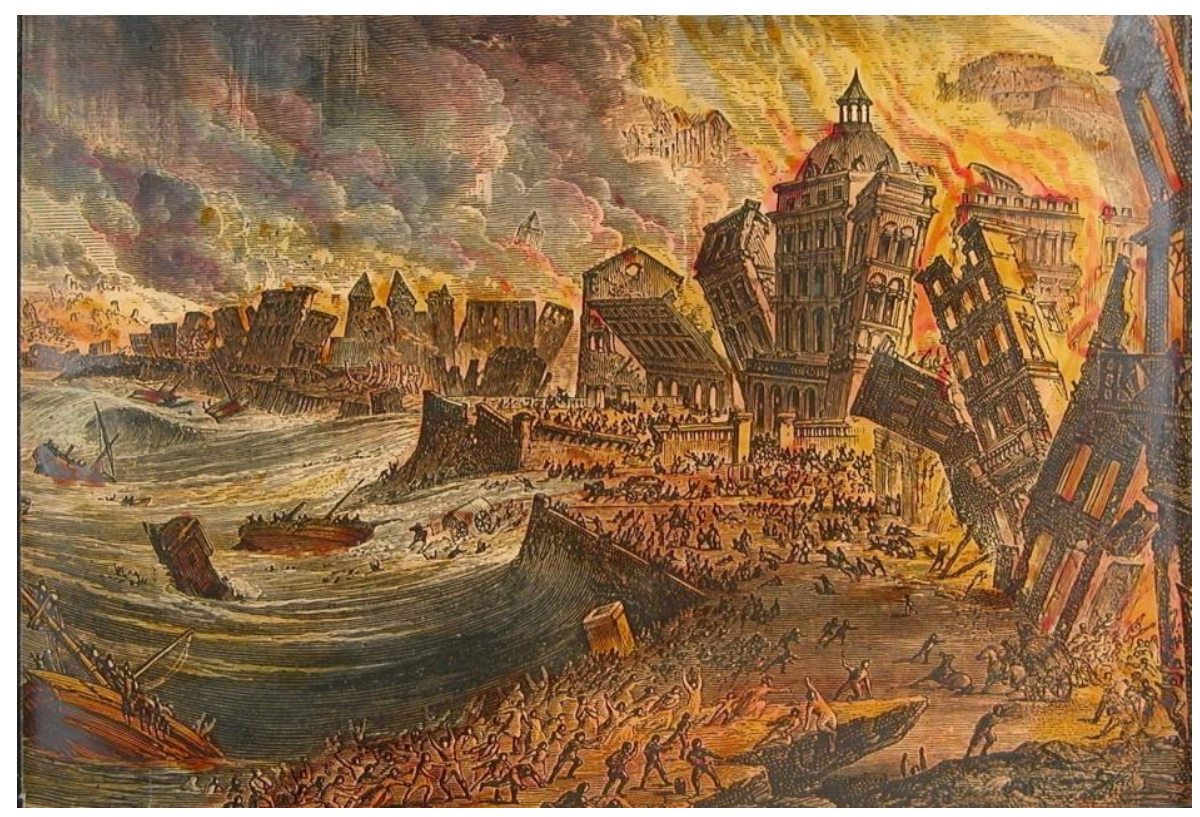

Figure 3. Gravure anonyme sur bois, $d u X I X^{e}$ siècle, peinte à la main, représentant le tremblement de terre dévastateur de Lisbonne survenu le $1^{\text {er }}$ novembre 1755.>

Source : http://zamane.ma/fr/un-tsunami-de-legende/

Ainsi, «l'évolution du risque en tant qu'objet d'étude consiste en une amélioration de sa gestion allant de pair avec un progrès des connaissances scientifiques » (Liéval, 2006 : 20). En parallèle, depuis la fin des années 1970, on observe une intolérance croissante aux risques, notamment dans les sociétés se revendiquant des valeurs écologiques. Suite au choc pétrolier de 1973, et sous l'influence de penseurs tels que Hans Jonas (1979), le mythe de la croissance infinie s'étiole tandis que la science jadis vectrice de l'idée de Progrès (positivisme, hygiénisme...) - est de plus en plus perçue comme un facteur de risque pour l'environnement ${ }^{16}$; « la science confère à l'homme des forces jamais encore connues, l'économie pousse toujours en avant dans une impulsion effrénée »(Jonas, 1979: 13). En Europe, en Amérique, au Japon, à partir de la première industrialisation (Verley, 1997), les explorations, l'usage du charbon, de l'acier, puis du nucléaire avaient pourtant contribué à améliorer le niveau de vie des populations. Ces avancées auraient-elles été possibles si leurs acteurs avaient d'emblée eu conscience de leurs conséquences humaines et environnementales ? Ne faudrait-il pas, alors, s’interroger sur la valeur de la prise du risque, voire sur sa nécessité ?

De nos jours, la prise de risque et son acceptation sont souvent décriées ${ }^{17}$; il n'est que de citer l'introduction du «principe de précaution ${ }^{18}$ dans le préambule de la Constitution de la République française, à la faveur de la révision constitutionnelle du $1^{\mathrm{er}}$ mars 2005. Ce principe interroge la notion d'incertitude, met en cause le statut du scientifique, les rapports entre recherche et politique, mais aussi le domaine juridique. En revanche, par exemple, il semble difficile de dresser une carte d'un risque non avéré. Au sein des recherches géographiques, ce «principe de précaution » est largement mobilisé à travers la pensée aménagiste, le domaine juridique et politique. Dans ce contexte, un questionnement peut contribuer à l'élaboration d'une réflexion géographique sur le risque : quelle place pour le

\footnotetext{
${ }^{16}$ Voir également Chassaigne (2008).

${ }^{17}$ Voir par exemple l'article 5 de la Charte de l'environnement : « Lorsque la réalisation d'un dommage, bien qu'incertaine en l'état des connaissances scientifiques, pourrait affecter de manière grave et irréversible l'environnement, les autorités publiques veillent, par application du principe de précaution et dans leurs domaines d'attributions, à la mise en œuvre de procédures d'évaluation des risques et à l'adoption de mesures provisoires et proportionnées afin de parer à la réalisation du dommage. " (Laquièze, 2012 : 549).

${ }^{18}$ Notons que le réalisateur Hubert Charuel, dans son long métrage intitulé Petit Paysan, sorti en août 2017, offre un regard acéré sur un agriculteur français confronté à la crise de la vache folle et à la brutalité du « principe de précaution ».
} 
géographe dans une réflexion transdisciplinaire sur le risque et son acceptation? Comment appréhender le risque et ses représentations dans une société de plus en plus médiatique et judiciarisée?

\section{Approcher le risque par le décloisonnement disciplinaire}

Au sens étymologique, la géographie est l'étude descriptive de la surface de la Terre. "Sans volonté d'exclure d'autres démarches notamment celles, nécessaires, d'inspiration à dominante naturaliste, la géographie prend sa place parmi les "sciences sociales de la nature". Cela permet de nouvelles rencontres, de nouvelles interactions au sein de l'enveloppe institutionnelle Géographie, qui demeure fortement présente dans les universités et qui, sans retour en arrière, peut devenir ou redevenir un cadre d'échanges intellectuels utile» (Lévy, 2000: 79). Ainsi, «la géographie "science sociale des territoires" doit enraciner l'environnement dans la nature et dans la société »(Deffontaines, 2004 : 234). L'étude des risques permet donc de s'affranchir des frontières, de dépasser les clivages et de réunir géographes physiciens et humains, par leur rapport à la nature, à l'environnement et à l'écoumène ${ }^{20}$ (Lacoste, 1982 : 9).

En France, depuis les années quatre-vingt, les risques naturels relèvent généralement de la géographie physique (Faugères, 1991: 180), mobilisant davantage l'étude des milieux (November, 2002 : 27). La thématique des risques sociaux et technologiques est quant à elle abordée par la géographie humaine (Ariano, 2008: 58). Science sociale, la géographie peut pourtant être définie comme l'étude de «l'espace des sociétés », ou encore de la dimension spatiale ${ }^{21}$ du social; soit la façon dont les sociétés établissent les distances entre leurs composants (individus, entreprises, États, ressources...). «Ce qui est premier en géographie sociale, c'est la société et non l'espace. Ce sont les mécanismes, les processus sociaux et sociétaux, le jeu des acteurs publics ou privés de toutes espèces. [...] Mais il est bien évident que l'espace à son tour rend bien compte de tout un jeu de variations qui renforcent ou atténuent les décalages et les tensions entre société locale et société globale » (Rochefort, 1983 : 13.). «La géographie doit être de son temps et contribuer à la prise de conscience collective et à la solution des problèmes de société ; (Frémont et al., 1984: 125). "Contribuer à décrypter l'organisation des sociétés, plus particulièrement leur organisation distribuée à la surface de la Terre, telle est l'ambition de la géographie sociale. » (Frémont et al., 1984 : 158). Il s'agit donc de dépasser l'approche analytique pour comprendre de quelle manière les sociétés jouent de l'espace pour s'organiser et se structurer. C'est pourquoi l'étude des risques doit relever d'une démarche de décloisonnement des disciplines.

Claude Lévi-Strauss (1952), Edgar Morin (1977, 2007), Marcel Roncayolo (2014), entre autres, ont insisté sur la mise à distance et la suspension dans l'analyse ${ }^{23}$. Il s'agit de comprendre les structures profondes qui échappent au regard, mais qui déterminent, beaucoup plus que ce que l'on croit, le développement des sociétés. Cette grille de questionnement, associée à la géographie, permet d'ajuster l'étude de l'objet - le territoire - et d'expliquer la présence d'un risque à un endroit et non à un autre. La géographie s'applique donc à déterminer ses causes, aussi bien naturelles qu'humaines, et ses conséquences multiples. Pour autant, l'enseignement dispensé, notamment dans le secondaire, fournit des connaissances éparses, qui deviennent affaire de spécialistes. Par exemple, l'étude d'un risque n'est

\footnotetext{
${ }^{20}$ Au sens de Berque (1986).

${ }^{21}$ L'approche dimensionnelle de la géographie sociale actuelle permet de « raisonner en termes de "dimension spatiale" permet de dépasser la fausse symétrie espace / société, [ $d$ ']éviter le piège de la réification de l'espace et d'affirmer clairement sa nature sociale » (Veschambre, $2006: 218$ ).

${ }^{23}$ Notons que les philosophes septiques de l'Antiquité grecque revendiquaient déjà l'épochè, la suspension de jugement. Voir Gill, Pellegrin (2006).
} 
enseignée que de manière diffuse, et ne fait l'objet d'aucun cours spécifique, si ce n'est dans le nouveau programme de géographie au lycée ${ }^{24}$.

De nos jours, ces savoirs cloisonnés tendent à être «ouverts et reliés » ${ }^{25}$. C'est là l'une des gageures des étudiants lors de leur arrivée à l'université, car la géographie du supérieur s'éloigne de celle du secondaire. Les intitulés des maquettes des diplômes universitaires de géographie parlent d'euxmêmes ; au travers des cours tels que «risques et sociétés », les étudiants abordent les grands thèmes environnementaux auxquels sont confrontés aménageurs et urbanistes, dans une perspective transdisciplinaire. Cette démarche surprend les étudiants, précédemment habitués à approcher les territoires par la cartographie (localisation, situation d'un territoire), l'économie (productions, activités) ou la géopolitique (relations internationales). Tous ces savoirs sont, bien évidemment, importants, mais ils doivent être relayés à des disciplines ignorées ou presque dans le secondaire, telles que la géomorphologie, la biopédologie, la climatologie ou la démographie, etc. Ces disciplines se révèlent indispensables à l'étude des risques.

Par ailleurs, ce qui a trait aux risques se partage entre les sciences et les arts. De fait, la littérature ${ }^{26}$, la peinture ${ }^{27}$, la musique ${ }^{28}$, la photographie ${ }^{29}$ ou le cinéma ${ }^{30}$ constituent aussi des sources de

\footnotetext{
${ }^{24}$ Les programmes du secondaire des années 1990 et 2000 intégraient peu le risque. Désormais, il n'est pas anodin que ce soit la géographie qui l'intègre le plus, notamment, depuis peu, via la question la prospective territoriale. Voir, par exemple, les programmes de géographie de fin de cycle 3 (classe de $6^{\mathrm{e}}$ ) et début de cycle $4\left(5^{\mathrm{e}}\right)$, dans le Bulletin officiel, arrêté du 9-11-2015 - J.O. du 24-11-2015 [URL : http://www.education.gouv.fr/pid285/bulletin_officiel.html?cid_bo=94708 ;

http://www.education.gouv.fr/pid285/bulletin_officiel.html?cid_bo=94717], consultés le 15 septembre 2017.

${ }^{25}$ Morin (2009) ajoute : "nous ne sommes pas dans une société de "la connaissance ", nous sommes dans une société " des connaissances séparées ». Ne croire qu'en des spécialités, c'est ne croire qu'en une vision de l'être humain borné et incapable de se poser des problèmes ».
}

${ }^{26}$ Exemples d'œuvres littéraires : Auster P., 1991, La musique du hasard ; Balzac H. (de), 1831, La Peau de Chagrin, La Recherche de I'Absolu ; Barjavel R., 1968, La Nuit des temps, 1943, Ravage ; Boyle T.C., 1995, America ; Cendrars B., 1925, L'Or, 1958, À I'aventure ; Chretien De Troyes, 1176, Yvain ou le chevalier au lion ; Dostoïevski F., 1866, le Joueur ; Freby F., 2005, Catastrophes naturelles et littérature ; Frison-Roche R., 1942 Premier de cordée ; Gide A., 1914, Les Caves du Vatican, le livre V (Lafcadio) ; GIONO J., 1984, Les trois arbres de Palzem ; Girard De Saint-Amant M. A., 1658, La Seine extravagante ; Goethe W. (von), 1808, Faust ; Hugo V., 1856, Les Contemplations, Livre III, 2, "Melancholia »; Huxley A., 1931, Le Meilleur des mondes ; Léry J. (de), 1578, Histoire d’un voyage fait en la terre du Brésil ; Maupassant G. (de), 1882, "Pierrot », dans le journal Le Gaulois ; Merle R., 1972, Malevil ; Mordillat G., 2005, Les vivants et les morts ; Ponson Du Terrail P., 1857, Rocambole ; Shelley M., 1818, Frankenstein ou le Prométhée moderne ; Simmons D., 2003, Ilium ; Stevenson R.L., 1886, L'étrange cas du docteur Jekyll et Mr Hyde ; Voltaire, 1756, Poème sur le désastre de Lisbonne. Pièces de théâtre : Corneille P., 1643, Cinna ; 6T Théatre, 2012, Tout, tout de suite ; Noville J-F., 2000, La supplication » Tchernobyl, chroniques du monde après l'apocalypse, d'après le roman d'Alexievitch S. édité en 1998.

${ }^{27}$ Exemples de compositions picturales : Bain M., 2005, Black Ship ; Beauvais H., 1856, L'Empereur visitant les inondés d'Angers en 1856 ; Freud L., 1993, Evening in the Studio ; Friesz O., 1940, Incendie des bacs de pétrole au Havre (500 000 t) ; Hokusai, 1830, La Grande Vague ; Kupka F., 1906, La remontée au jour des revenants de Courrières ; Pfauntz G.C., 1755, Représentation du désastre de Lisbonne ; Gericault T., 1816, Le radeau de la Méduse ; Moser H., 1570, Inondation de la région d'Anvers ; Pinchon R. A., 1907, Les prairies inondées ; Provost A., 1842, Catastrophe ferroviaire de Meudon ; Illustrations dans le Petit Journal, mars 1906, Catastrophe minière de Courrières ; Sisley A., 1876, L'inondation à Port-Marly ; Stroberle J.G., Sismo de 1755 ; Tsykalov D., 2005, Skull ; Turner W., 1810, Avalanche dans les Grisons ; Van Heil D., 1662, Incendie de la poudrière d'Anvers ; ajoutons à cette liste non exhaustive les gravures anonymes sur le tremblement de terre de Lisbonne de 1755.

\footnotetext{
${ }^{28}$ Exemples de compositions musicales : Debussy, 1905, La mer (opus L109) ; Cali, 2005, "La fin du monde pour dans $10 \mathrm{mn}$ ", extrait de l'album Menteur ; Thiefaine H. F., 1979, Alligator 427; Mickey 3D, 2003, Respire ; Noir Désir, 2001, Le grand incendie Vivaldi A., 1725, Le quattro stagioni, Opus 8, n¹-4, qui ouvrent le recueil « La confrontation entre l'harmonie et l'invention ».

${ }^{29}$ De nombreuses collections photographiques, privés et publics, existent sur les risques. Citons par exemple, les expositions photographiques de Brueggemann F., 2011, "Au-delà d'une possible représentation - la photographie japonaise et la bombe atomique »; Clover S., 2012, 1906 + Today: The Earthquake Bend ; Estebe C., 2012, « Le premier Godzilla (1954), le monstre de la (c) 2018 ISTE OpenScience - Published by ISTE Ltd. London, UK - openscience.fr 
connaissances aussi bien en ce qui concerne les représentations du risque que les conséquences des événements. Parfois critiquées ${ }^{31}$, ces sources fournissent cependant des données importantes sur l'habitat humain, les innovations, les outils et les moyens mis en place. Elles expriment les diverses représentations et les perceptions sociales du risque, qu'il soit naturel, industriel ou technologique. La mémoire des catastrophes est également à prendre en compte, à travers les commémorations ou les inaugurations de mémoriaux ${ }^{32}$, qui rappellent la permanence du risque sur les sociétés humaines. Ces monuments peuvent être compris comme des actes résilients destinés à consolider une communauté psychologiquement affectée par une catastrophe. C'est le cas de la réalisation pédagogique du collège Stendhal de Toulouse à la suite de l'explosion de l'usine AZF en septembre $2001^{33}$, ou encore du mémorial de Malpasset à Fréjus, construit en 2009 en souvenir de la vague géante et meurtrière de décembre 1959.

catastrophe », France Culture, "La Catastrophe dans l'imaginaire cinématographique », 14 août 2012 ; Vartanian I., 2011, " Quelles images après le tremblement de terre et le tsunami ? "; puis l'exposition " Climat, à nos risques et périls ! Les Lyonnais face aux caprices du climat depuis le Moyen-âge ", aux Archives municipales de Lyon, du 10 octobre 2012 au 30 mars 2013 , retrace à partir de peintures datant de la fin du Moyen-âge, et de photographies réalisées dès le XIXe $\mathrm{S}$. les aléas du climat à Lyon.

${ }^{30}$ Concernant le septième art, les exemples sont nombreux : Bayona J.A., 2012, The impossible ; Bertrand Y. H., 2010, Home ; Boyle D., 2010, 127 hours ; Burton G., 1998, Aftershocks ; Clouzot H-G., 1953, Le Salaire de la peur (adapté du roman du même nom D’arnaud G.) ; Emmerich R., 2004, Le jour d'après ; Elikann L., 1990, Séisme ; Emmerich R., 2009, 2012 ; Fankhauser A., 2009,2012 : Supernova ; Feng X., 2010, Tremblement de terre à Tangshan ; Fouche A., 2011, Memories Corner ; Guillermin J., 1974, La tour infernale ; Guzzanti S., 2010, Draquila. L'Italie qui tremble ; Higuchi S., 2006, Sinking of Japan ; Latt D. M., 2009, Secousse sismique ; Lewis R. M., 1974, The Day the Earth Moved ; Lopez N., 2013, AfterShock ; Martinelli R., 2001, La folie des hommes ; Marton A., 1965, Quand la terre s'entrouvrira ; Mizuta N., 2008, Code 252 : Signal de détresse ; Negulesco J., 1955, La Mousson ; Niccol A., 1997, Bienvenue à Gattaca ; Pellerin J., 2000, Daybreak, le métro de la mort ; Pepin R., 2000, Secousses à Los Angeles ; Ohmori K., 1980, Cataclysme force 7.9 ; Reeves S., 1959, Les derniers jours de Pompéï ; Robson M., 1974, Tremblement de terre ; Sauper H., 2004, Le cauchemar de Darwin ; Shavelson M., 1960, C'est arrivé à Naples ; Sears F. F., 1957, The night the world exploded ; Shear B., 1972, Short walk to daylight ; Soderbergh S., 2000, Erine Brockovich ; Spielberg, 2001, A.I. Artificial Intelligence ; Takacs T., 2004, Earthquake ; Wachowski A., Wachowski L., 1999, Matrix (trilogie).

${ }^{31}$ Cette réflexion fait suite à la lecture d'un article d'Edgar Morin (2009) dans lequel le sociologue et philosophe défend l'idée d'une culture qui relie nos connaissances éparses.

${ }^{32}$ Sur l'histoire et la mémoire voir notamment Nora (1997).

${ }^{33}$ L'usine chimique AZote Fertilisants classée Seveso : site industriel présentant des risques d'accidents majeurs sur lequel était maintenu un haut niveau de prévention. 


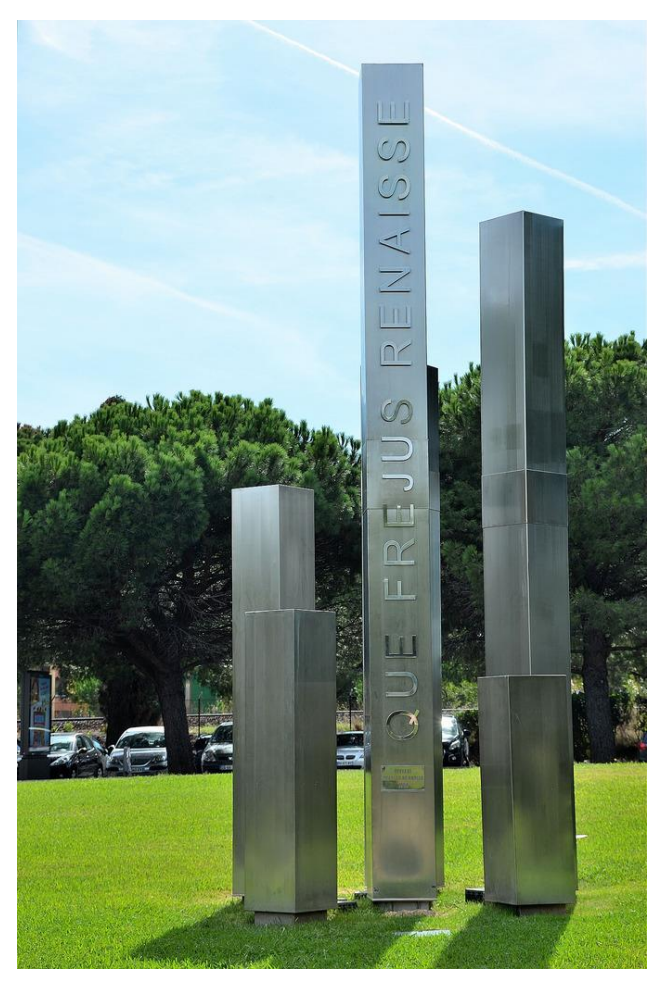

Figure 4. Le mémorial de Fréjus, réalisé par le sculpteur Michel Mourier, dévoilé le 2 décembre 2009, à l'occasion du $50^{\circ}$ anniversaire de la catastrophe, sur lequel figure les noms des victimes et le vœu du général de Gaulle lors de sa visite du 9 octobre 1961, "Que Fréjus renaisse! ».

Source : Patrick (2015)

A ces exemples de monuments s'ajoutent les créations d'artistes, comme le peintre Raymond Beauduy qui, suite au séisme de 2010 en Haïti, place au centre de la toile des maisons en ruine ou vacillantes et des corps gisant au milieu des rues, dans des tons gris et tristes, ce qui amène à penser que l'art haïtien a perdu une part de sa naïveté ${ }^{34}$. Citons aussi le travail d'écriture de Dany Laferriere, qui dans Tout bouge autour de moi, raconte son expérience du séisme qui a ravagé l'île ${ }^{35}$. Cette mémoire des catastrophes ne se limite pas aux désastres naturels; elle englobe aussi le tourisme macabre $^{36}$ ou les violences humaines de plus en plus appréhendées comme des risques (risque de guerre, risque terroriste $\left.{ }^{37} \ldots\right)$.

Ainsi, les risques, comme tout ce qui relève des sociétés humaines - villes, modes de vie, innovations - constituent des sources inépuisables d'inspirations artistiques et scientifiques. La géographie ne peut donc pas se réduire aux savoirs transmis par le langage ; l'art, par le patrimoine matériel et immatériel, renseigne aussi sur les habitudes, les coutumes, les aménagements et les problèmes des sociétés (pastorales ou urbaines) qui relèvent de la thématique des risques. Par exemple, dans le cas de Venise, les œuvres picturales de Véronèse, Canaletto et Bellotto ont permis d'évaluer quantitativement le risque de submersion historique de la ville (Camuffo, 2010). Les chansons et danses folkloriques régionales sont aussi des témoignages, des révélateurs temporels et sociologiques

\footnotetext{
${ }^{34}$ Voir l'article de Sabourin (2010).

${ }^{35}$ Cf. site internet : http://memoiredencrier.com/tout-bouge-autour-de-moi-dany-laferriere/, consulté le 27 août 2017.

${ }^{36}$ Le tourisme macabre est centré « sur la visite des sites de mort et de dévastation. [...] II participe [...] à la patrimonialisation des espaces affectés par la catastrophe, [...] il met en lumière les enjeux politiques, sociaux, culturels liés à l'interprétation de l'évènement et aux discours sur la reconstruction de la ville (Hernandez, $2008: 61$ ).

${ }^{37}$ Par exemple, le mémorial newyorkais du 11-Septembre matérialise la mémoire des victimes des attentats de 2001.
} 
qui renseignent sur les us et coutumes face aux aléas climatiques ; c'est en cela que l'on peut parler d'un langage artistique.

Il faut donc relier ces savoirs pour les rendre intelligibles. C'est pourquoi il pourrait être judicieux d'introduire l'analyse de risques propres à notre civilisation - comme les aménagements des cours d'eau ou des montagnes - dans des cours d'économie ou de sociologie qui, traditionnellement, les ignorent. Par exemple, les risques en montagne sous-entendent, outre les typologies classiques, les mesures de protection, une analyse des activités humaines : toutefois, un paradoxe peut être souligné, celui que les êtres humains soient plus nombreux en hiver, dans des territoires où finalement l'aléa est plus fort. A ce sujet, les géographes Germaine et Paul Veyret, en acquérant une intelligence de la vie des hommes dans la réalité du quotidien, avaient inscrit, dans le hall d'entrée de l'Institut de Géographie Alpine, de Grenoble, la maxime «Alpes, terre de labeur ; terre de grandeur »; « un tel message dépasse l'instant et se perpétue dans le temps» (Billet, 1988: 113). De même, les implantations de sites manufacturiers dans les pays industriels, depuis le $\mathrm{XIX}^{\mathrm{e}}$ siècle, ou les usages des outils de planification par les aménageurs et les élus locaux doivent être intégrés à une approche géographique des risques dans leur globalité. S'intéresser aux risques (naturels, industriels, technologiques) amène à émettre deux constats: les risques apparaissent, désormais, comme une question centrale en géographie ; la thématique des risques transcende les frontières traditionnelles des disciplines.

Le géographe semble bien placé pour relier et synthétiser les différentes approches du risque. Il peut appréhender le risque dans ses dimensions physiques et humaines par l'étude des interactions entre l'homme et son milieu. Pourtant, à la lecture du Traité des nouveaux risques (Godard, 2002), rédigé par des économistes, ingénieurs ou spécialistes de l'assurance, l'on constate que les géographes restent à l'écart de la réflexion développée. De même, un ouvrage intitulé Pour un catastrophisme éclairé. Quand l'impossible est certain (Dupuy, 2002) propose une approche historique de la question en s'appuyant sur les travaux de philosophes, d'ingénieurs, d'économistes et de sociologues, sans inclure ceux de géographes. Autrement dit, les risques apparaissent comme une question majeure en géographie, tandis que la géographie n'apparaît pas centrale dans l'étude des risques. On peut donc se demander si les géographes ne seraient pas handicapés par leur propre culture disciplinaire, qui les empêcherait de cerner les enjeux dans toute leur dimension.

Dans l'enseignement de la géographie, la célèbre équation « Risque = Aléa x Vulnérabilité » est une sorte de formule pédagogique simplifiée envers laquelle de nombreux géographes prennent leur distance $^{41}$. Beaucoup d'universitaires définissent le risque par une équation, au lieu d'une fonction de l'aléa et de la vulnérabilité. Le risque englobe en fait l'aléa, la vulnérabilité, les enjeux et la capacité de réponse ou de résilience. D’ailleurs, des auteurs, comme Dauphine (2005), substituent à cette traditionnelle équation la formule suivante : «Risque $=\mathrm{f}$ (aléa, vulnérabilité) », où «f » est une fonction, une relation qui dépend du contexte. Au sein de cette nouvelle approche géographique, la gestion du risque passe d'abord par une gestion de la vulnérabilité, prenant en compte la population locale et ses spécificités. Gaillard (2003) démontre que l'intégration des facteurs ethnoculturels est un enjeu majeur pour la gestion des risques et des catastrophes naturelles, les peuples autochtones et les minorités ethniques restant les parents pauvres de la gestion des risques. C'est le cas des populations autochtones des îles Andaman abandonnées par les autorités indiennes, après le tsunami du 26 décembre 2004 dans le golfe du Bengale (Gosh, 2005). En ce sens, l'approche fondée sur la vulnérabilité apparaît véritablement géographique, puisqu'à l'interface entre nature et société.

\footnotetext{
${ }^{41}$ L'ensemble de cette réflexion fait suite à une discussion, en septembre 2011, avec Cécile Liéval (2006), docteure en géographie, suite à la soutenance de sa thèse, en 2013, sur les nouveaux risques, Innovation technologique et risques insaisissables : une territorialisation impossible ?, sous la direction du Professeur Soubeyran O., à l'Institut de Géographie Alpine.
} 
Pour Pigeon (2003a) la décomposition du risque en deux paramètres, l'aléa et la vulnérabilité, serait à l'origine d'un éclatement de la géographie des risques en différentes sous-disciplines, selon l'aléa étudié. La géographie de la santé traiterait des risques sanitaires, la géographie économique des risques technologiques, etc. Or, il faut s'intéresser aux relations entre aléa et vulnérabilité : ce ne sont pas les concepts en eux-mêmes qui sont à remettre en cause, mais les relations qu'ils entretiennent. Tout se passe comme si la géographie traitait des risques de façon simplifiée, en présentant des relations à sens unique. Ces approches apparaissent donc à la fois dominantes et limitées, car segmentées, surtout dans le cas d'une approche par type de risque : «identifier des freins à une évolution revient aussi à révéler d'autres conditionnements, apparemment inverses puisque favorisant l'évolution » (Pigeon 2003a). Cependant, les regroupements par espace peuvent induire une division des risques proche de la typologie: quand on étudie le risque en montagne, ce n'est pas pour parler du risque technologique (Dauphine, 2005). Le processus d'urbanisation est au cœur de ce renouvellement épistémologique, qui relie géographie fondamentale et géographie appliquée. Ici, les géographes sont influencés par la mutation des peuplements. Une inondation a une portée spécifique en milieu urbain : d'une part, du fait du grand nombre de personnes concernées, qui accroit la vulnérabilité ; d'autre part, en raison de l'imperméabilisation des sols. L'urbanisation génère donc deux évolutions : celle du risque et de ses nouveaux aléas et celle de l'étude du risque. Ainsi, l'urbanisation incite à adopter une lecture plus systémique des risques. En ce qui concerne l'inondation, il n'y a pas seulement conjonction d'un aléa extérieur et d'une vulnérabilité passive : le milieu urbain modifie l'aléa auquel il est exposé. En sortant du primat du risque naturel, on identifie de nouveaux aléas, ce qui amène à insister sur la notion de vulnérabilité. En effet, il y a trente ans, l'existence de risques naturels était largement acceptée par la société. De nos jours s'est engagée une réflexion sur la notion de risque, distinguée de l'aléa, à laquelle s'ajoute une réflexion sur le rôle et les effets des activités humaines.

Face au caractère réducteur du couple aléa et vulnérabilité pour la compréhension du phénomène d'urbanisation, Patrick Pigeon propose la solution de la «systémique », qui substitue à ces deux notions celle d'endommagement. L'auteur insiste sur la multiplication des risques qu'il faut ensuite gérer : un endommagement imputé à un aléa ou à un type d'aléa ne permet pas, à lui seul, de comprendre le risque. Celui-ci tient autant à une mutation du peuplement qu'à une éventuelle évolution des aléas hydrométéorologiques. La réflexion sur le risque semble donc largement perfectible, l'approche transdisciplinaire restant encore inaboutie. C'est pourquoi nous proposons d'aborder certaines dimensions du risque à même de la développer, en questionnant le rapport au risque des sociétés contemporaines.

\section{Prévention et représentations du risque}

De nouvelles problématiques émergent et mettent en jeu le couple «sociétés / risques »: elles impliquent un renouvellement des modes de gestion du risque. Des solutions ont ainsi été mises en place en France, telles que le transfert de compétences de l'État aux échelons locaux, pour faire concorder les initiatives avec la réalité du terrain. La territorialisation des politiques publiques se fait de plus en plus forte et des expériences de développement local émergent : les communes concernées sont consultées lors de l'élaboration du Plan de Prévention des Risques (PPR); l'échelon départemental se voit attribuer la compétence de la gestion et de la prévention des risques naturels ; des programmes régionaux d'aménagement concernent, pour partie ou en totalité, les infrastructures, les modes de production, la répartition des hommes et des activités, tels que les grands aménagements fluviaux destinés, entre autres, à améliorer la navigation fluviale, maîtriser l'irrigation et les risques d'inondation.

Les littoraux français, qui connaissent actuellement une urbanisation rapide, offrent un exemple de cette territorialisation du risque. Représentant $4 \%$ du territoire, ils abritent $10 \%$ de la population 
(CGET, 2014 : 24), et devraient accueillir, en 2040, plus de quatre millions d'habitants ${ }^{46}$. Or, le recul entrainé par l'érosion concerne un quart des côtes métropolitaines; a contrario, seul un dixième des littoraux est en extension (Colas, $2006: 1$ ). Malgré les nombreux ouvrages de défense réalisés pour limiter cette érosion, ou encore endiguer l'avancée des dunes, ces proportions ne varient pas depuis vingt ans; des procédures de péril sont d'ailleurs mises en œuvre dans les territoires les plus vulnérables ${ }^{47}$. Ces nouvelles politiques de gestion du risque ne semblent donc pas entièrement efficientes, d'autant que l'urbanisation contemporaine ne va pas sans paradoxes.

L'habitat tend en effet à se concentrer sur des territoires à risques - comme les littoraux ${ }^{48}$ - ce qui accroît la vulnérabilité des sociétés, tout en générant une demande croissante de limitation des risques. Depuis les années soixante, la croissance démographique, conjuguée à l'héliotropisme, à l'accélération de l'exode rural et au développement touristique, a entrainé une densification urbaine des vallées et des littoraux. Celle-ci se matérialise par l'implantation de nouveaux lotissements, de supermarchés et de zones d'activités construites en zones inondables ${ }^{49}$. Pour limiter les inondations, des digues ont souvent été mises en place. Cependant, elles n'ont pas complètement empêché la montée des eaux. Avec ce type d'équipement, il est donc facile de mesurer «l'avant», les plus hauts niveaux atteints par les crues historiques, et de constater «l'après », l'utilité des digues en question. Il s'agit bien d'un risque connu, fréquent et régulier à l'échelle des temps géologiques. La prévention ne consiste donc pas en la suppression de l'aléa, mais en la diminution de la vulnérabilité (Pigeon, 2003b). Ici, on observe le traitement d'un risque territorialisé, où l'articulation entre compréhension, prédiction et prévention, semble fonctionner et répondre aux demandes angoissées des habitants. Ce dernier point est à considérer, car le risque se définit aussi par sa perception et les représentations que s'en font les populations concernées.

A ce sujet, la prise de conscience du risque augmente avec la médiatisation des catastrophes: ouragan Katrina de 2005 dans l'océan Atlantique Nord, séisme de 2011 sur la côte Pacifique du Tohoku (Japon), ou encore cyclone Irma à Saint-Martin en septembre 2017. Les réactions des autorités à ces catastrophes suscitent parfois des polémiques ${ }^{50}$. Celles-ci renvoient pourtant à des risques collectifs majeurs $^{51}$, certes, mais anciens, que l'on peut considérer comme «classiques ». D'ailleurs, qu'ils soient «plus discrets, spatialement diffus, étalés dans le temps, [...] par leurs effets de gênes et de nuisances [ils] affectent la santé publique et sont peut-être, sur le long terme, causes d'autant de pertes humaines que les grandes catastrophes »(Dubois-Maury, 2002: 191). Mais ces catastrophes

\footnotetext{
${ }^{46}$ Voir site Internet de l'observatoire national de la mer et du littoral, « Démographie, occupation du sol et logement »: http://www.onml.fr/chiffres-cles/cadrage-general/demographie-occupation-du-sol-et-logement/], consulté le 28 août 2017.

${ }^{47}$ Cf. article L. 511-1 à L. 511-6 et les articles R. 511-1 à R. 511-12 du code de la construction et de l’habitation.

${ }^{48}$ En France métropolitaine, les territoires urbanisés couvrent 22,8\% des terres situées à moins de $250 \mathrm{~m}$ des côtes reculant. La construction de logements est très forte dans les communes littorales : $10 \%$ des surfaces de logements y sont construits sur seulement 4\% du territoire. Le quart du littoral métropolitain (24\%) subit l'érosion et le trait de côte recule, soit $1720 \mathrm{~km}$ sur 5500 $\mathrm{km}$ de côtes, alors que $44 \%$ du littoral sont stables et seulement $10 \%$ sont en extension. Ce constat est le même en Europe où $20000 \mathrm{~km}$ de côtes, soit $20 \%$ du littoral, s'érodent ; une superficie de $15 \mathrm{~km}^{2}$ de terres est perdue ou fortement endommagée annuellement. (Colas, 2006).
}

${ }^{49}$ En France, entre 1999 et 2006, 100000 logements ont été construits en zone inondable par un cours d'eau, dans des communes de 10000 habitants dès lors que le respect de certaines conditions l'autorisait. De nos jours, le risque inondation concerne plus de 13000 communes dont 300 grandes agglomérations ; 17 millions d’habitants vivent dans des territoires vulnérables et $40 \%$ des emplois sont directement concernés par le risque d'inondation (Lecomte, 2015 : 3).

${ }^{50}$ Cf. article de Pietralunga, Bonnefous (2017).

\footnotetext{
${ }^{51}$ Face à ce type de catastrophe que l'on aurait tendance à qualifier de risque naturel ou climatique, de nombreux scientifiques ont mis en avant les responsabilités humaines.
} 
sont vécues comme des événements extraordinaires et inacceptables. L'émotion qu'elles suscitent est parfois planétaire. Par exemple, en décembre 2004, le tsunami qui a frappé les littoraux d'Asie du SudEst a entraîné la mort de milliers de personnes originaires des quatre coins du monde et la destruction d'une grande partie des rivages septentrionaux de l'océan Indien (Ozer, De Longueville, 2005 : 1). Le grand nombre de victimes et leurs origines variées expliquent sa médiatisation et la solidarité internationale qu'il a suscité (Debarbieux, 2005). Suite à cette catastrophe, «ces dernières années, des changements sont apparus dans l'environnement politique et social de l'aide humanitaire » (Reymond et al., 2017 : 13). La présence de touristes occidentaux a probablement accentué l'identification aux victimes du tsunami. Les images amateurs prises par ceux-ci, comme les reportages télévisés ont ému de nombreuses personnes à travers le monde ${ }^{54}$. Pourtant, «sur le plan scientifique strict, il s'agissait d'un événement d'ampleur moyenne comparé à d'autres vagues survenues dans le passé sur d'autres secteurs » (Pascual et al., 2009 : 80).

On peut par ailleurs s'interroger sur l'influence de la fiction grand public dans les représentations médiatiques de la catastrophe. L'imaginaire de la catastrophe s'affirme, dans le cinéma grand public, à partir des années soixante-dix, marquant un certain désenchantement vis-à-vis du progrès technologique, par exemple avec Airport de George Seaton (1970), La tour infernale de John Guillermin et Irwin Allen (1974), L'aventure du Poséidon de Ronald Neame (1972), Mondwest de Michael Crichton (1973). Puis, vient la naissance d'une conscience écologique, de Soleil vert de Richard Fleisher (1973), à Twister de Jan de Bont (1996), en passant par La planète des singes de Franklin D. Schaffner (1968), Le Mystère Andromède de Robert Wise (1971). Cet imaginaire se prolonge aujourd'hui dans certaines séries télévisées comme Jericho (2006) et Walking dead (2010). Les vidéos amateurs pourraient avoir été contaminées par l'esthétique apocalyptique d'un cinéma aux titres aussi évocateurs qu'Armageddon, deMichael Bay (1998), ou Le jour d'après, de Roland Emmerich (2004). Ces images anxiogènes circulent dans un monde hyperconnecté, où les réseaux sociaux répercutent les angoisses contemporaines. L'émotion apparait donc comme une dimension importante dans l'appréhension du risque, perçu alors avec davantage d'acuité. Il faudrait aussi s'interroger sur la vogue du survivalisme qui a essaimé depuis les Etats-Unis et connait un certain succès sur le net ou dans des émissions grand public comme Man vs Wild. Les figures de cette communauté proposent des techniques de survie : loin de refuser le risque, ils prétendent l'anticiper pour mieux l'affronter. A ce titre, des ouvrages comme ceux du très controversé Piero San Giorgio particulièrement actif sur Internet - semblent trouver leur public.

Ce constat amène à reconsidérer la prise de conscience du risque par les sociétés humaines. Il n'y a pas de risque ni de catastrophe sans conscience de ceux-ci (Guénard, Simay, 2011); la société peut créer du risque en considérant qu'un événement aléatoire, jadis intégré à la vie ordinaire d'une collectivité, devient intolérable ${ }^{57}$ (Beck, 2001). Au cours du temps, les sociétés humaines ont, en effet, cherché à se prémunir de ce qu'elles percevaient comme des menaces (inondation, éruption volcanique, foudre, avalanche, épidémie...), que ce soit par des offrandes aux dieux ${ }^{58}$ ou, plus récemment, par la souscription à des assurances. Le développement de celles-ci reflète une prise de conscience généralisée du risque, en témoignent l'apparition des Catastrophes Bonds ${ }^{59}$, qui intègrent le

\footnotetext{
${ }^{54}$ Voir aussi la scène inaugurale du film Au-delà, réalisé par Clint Eastwood, en 2010, dont la vague y est reconstituée de façon spectaculaire.

${ }^{57}$ Par exemple, en matière de sécurité routière, de consommation alimentaire, d'alcool, de tabac...

${ }^{58}$ Pour une réflexion sur les thématiques de la fin du monde et des catastrophes, dans une perspective interdisciplinaire, voir Bornet et al. (2012).

59 Une " Catastrophe Bond » ou « CAT Bond » est une obligation dont le versement des intérêts et le remboursement du principal dépendent de la survenance d'une ou plusieurs catastrophes naturelles, tels qu'une tempête, un tremblement de terre ou une pandémie. Ce type d'obligation est émis par des compagnies d'assurances ou de réassurance, en vue de la titrisation de leurs (c) 2018 ISTE OpenScience - Published by ISTE Ltd. London, UK - openscience.fr 
rapport de chaque ville aux risques naturels et industriels. La prime d'assurance devient plus coûteuse en zone sismique pour les agglomérations dépourvues d'une réglementation en urbanisme ; certaines villes renoncent alors à s'assurer. Ici, l'assurance limite la prise de risque au lieu de la favoriser. En admettant que le risque soit la conjonction de l'aléa et de l'enjeu, ce système devrait entraîner une augmentation progressive des primes d'assurances et une sanction plus importante des risques pris au regard de la loi. Cette monétarisation du risque accroit la judiciarisation de la société et pourrait, à terme, la paralyser par le refus de toute prise de risque. Si ces craintes peuvent sembler extrêmes, le poids actuel des compagnies d'assurance (publiques ou privées) est indéniable et symptomatique : une plus grande sensibilité aux risques caractérise l'époque ; la nature de ceux-ci changerait alors moins que leurs perceptions.

\section{Conclusion}

$\mathrm{Au}$ carrefour de plusieurs disciplines scientifiques, la géographie peut, au moyen d'une approche essentiellement territoriale, contribuer à l'étude des risques. Dans cette perspective, la dimension subjective apparait incontournable : le risque relève d'abord d'une perception, matérialisée ensuite dans les processus d'occupation, d'aménagement et d'appropriation des territoires.

A l'issue de cette réflexion exploratoire, plusieurs questions se font jour : la société de demain serat-elle une société du moindre risque ? Une société qui se prémunit autant des risques ne risque-t-elle pas de devenir de plus en plus vulnérable? Quels impacts sur les territoires et sur les politiques publiques?

\section{Bibliographie}

Gilard O., Mesnil J.J., (1994), «Le risque d'inondation : analyse de la crue de Vaison-la-Romaine, quelques réflexions sur le risque d'inondation », Bulletin de l'AIGREF, n²4, $12 \mathrm{p}$.

Akoun A., (2001) Dictionnaire de sociologie. Ed. Larousse, p. 45.

Alois F., (2012), «Vers une nouvelle solidarité », dans Lettre de Taizé, n²74, édition spéciale, p. 1.

Ariano S., (2008), Pour une étude géographique du risque : les zones humides. Application à l'analyse du delta du Po. Thèse de doctorat en Géographie, sous la direction de Micheline Hotyat et de Marina Bertoncin, Soutenue en 2008, Paris 4, $260 \mathrm{p}$.

Becerra S., (2012), «Vulnérabilité, risques et environnement: l'itinéraire chaotique d'un paradigme sociologique contemporain », VertigO - la revue électronique en sciences de l'environnement, Vol. 12, n ${ }^{\circ}$, mai 2012, mis en ligne le 29 mai 2012, [URL: http://vertigo.revues.org/11988; DOI : 10.4000/vertigo.11988], consulté le 04 septembre 2017.

Beck U., (2001), La société du risque. Sur la voie d'une autre modernité, Ed. Flammarion, Paris, 521 p.

Berque A., (1986), Le Sauvage et l'Artifice. Les japonais devant la nature, Paris, Gallimard, p. 66 et sequitur.

Billet J., (1988) «In memoriam. Paul Veyret (1912-1988)», Revue de géographie alpine, Hommage de l'Académie Delphinale - Extraits (séance du 26 mars 1988), tome 76, n², pp. 99-119. [URL : www.persee.fr/doc/rga_00351121_1988_num_76_2_2703], consulté le 12 février 2016.

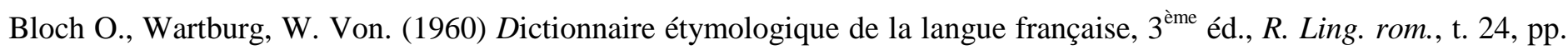
288-289.

Bornet P., Clivaz C., Durisch-Gauthier N., Hertig P., Meylan N., (2012), La fin du monde. Analyses plurielles d'un motif religieux, scientifique et culturel. Ed. Labor et Fides, Coll. Religions et modernités, 249 p.

risques liés à la survenance d'événements « catastrophes ». Les Assurances Bonds sont faciles à financer pour les pays développés, mais moins pour les autres. 
Bouhdiba S., (2014), «Lisbonne, $1^{\text {er }}$ novembre 1755 : un hasard? Au cour de la polémique entre Voltaire et Rousseau », Carnet de recherche hypothèses "Presque Partout», mis en ligne en octobre 2014, [URL : http://presquepartout.hypotheses.org/1023], consulté le 11 septembre 2017.

Bourdin A., (2003) «La modernité du risque », Cahiers internationaux de sociologie, vol. 114, n ${ }^{\circ}$, pp. 5-26 [URL: https://www.cairn.info/revue-cahiers-internationaux-de-sociologie-2003-1-page-5.htm], consulté le 17 juin 2018.

Bouyssou G., (2016), «Tyrannis, basileia, imperium, les composantes militaires des légitimités politiques dans le monde gréco-romain (Ve siècle avant-IVe siècle après J.-C.) », Revue internationale d'Histoire Militaire Ancienne, Klincksieck, pp. 3-32.

Bresson A., (2008), L'économie de la Grèce des cités. II. Les espaces de l'échange, Paris, Armand Colin, 334 p.

Brunet R., (2006), Les Mots de la géographie, Ed. La Documentation française, Reclus, 520 p.

Camuffo D., Bertolin C., (2010), «Le risque de submersion historique à Venise et le Projet MOSE », présentation au Colloque Les littoraux à l'heure du changement climatique, Rochefort, Brouage, La Rochelle, 18-20 novembre 2010.

CGET, (2014), «Rapport 2014. Démographie», L'observatoire des territoires, partie 1, fiches thématiques, A Démographie, 2. Logement », [URL: http://www.observatoire-des-territoires.gouv.fr/observatoire-desterritoires/sites/default/files/documents/2_Fiches_thematiques_Demographie.pdf], consulté le 28 août 2017.

Chassaigne P., (2008), Les années 1970. Fin d'un monde et origine de notre modernité, Ed. Armand Collin, 368 p.

Colas S., (2006), «Un quart du littoral recule du fait de l'érosion», Institut français de l'environnement, $\mathrm{n}^{\circ} 113$, septembre 2006, pp. 1-4.

Cooley C. H. (2016) « La Conscience sociale (traduction par Baptiste Brossard) », Sociologie, n², vol. 7, mis en ligne le 26 septembre 2016 [URL : http://journals.openedition.org/sociologie/2775], consulté le 22 juin 2018.

D’Ercole R., Pigeon P., (1999), «L'expertise internationale des risques dits naturels : intérêt géographique », dans Annales de géographie, n 608, pp. 339-357.

Dauphine A., (2005), Risques et catastrophes. Observer, spatialiser, comprendre, gérer, Ed. Armand Colin, Paris, 288 p.

Debarbieux B., 2005, «La première catastrophe naturelle médiatisée ?», Le Soir, Bruxelle6 janvier 2005, 13.

Deffontaines J.-P., (2004), «Une géographie traversière. L'environnement à travers territoires et temporalités », dans Natures Sciences Sociétés, Lectures : Comptes rendus, vol. 12, pp. 231-245.

Delumeau J., (1978), La Peur en Occident XIVe-XVIIe siècles, Ed. Fayard, Paris, 485 p.

Delumeau J., (1983), Le Péché et la peur. La culpabilisation en Occident (XIIIe-XVIIIe siècle), Ed. Fayard, Paris, 741 p.

Delumeau J., (1987), Les Malheurs des temps. Histoire des fléaux et des calamités en France, Ed. Larousse, Paris, 519 p.

Delumeau J., (1989), Rassurer et protéger. Le sentiment de sécurité dans l'Occident d'autrefois, Ed. Fayard, Paris, PAGES.

Dollo C., Lambert J-R., Parayre S., (2017), Lexique de sociologie. Ed. Dalloz, $5^{\mathrm{e}}$ édition, Paris, p. 325.

Dubois-Maury J., Chaline C., (2002), Les Risques urbains, Ed. Armand Colin, Coll. « U », Paris, 208 p.

Duméry H., (2017), Encyclopadia Universalis, définition de «Théodicée », [URL : http://www.universalisedu.com/encyclopedie/theodicee/], consulté le 11 septembre 2017.

Dupuy J.P., (2002), Pour un catastrophisme éclairé. Quand l'impossible est certain, Paris, Seuil, 216 p.

Faugères L., (1991), «La géo-cyndinique, géoscience du risque », Bulletin de l'Association de Géographes français, 3 , pp. 179-193.

Ferrier J.-P., (1998), Le contrat géographique ou l'habitation durable des territoires. Antée 2, Lausanne, Payot, 251 p.

Frémont A., Chevalier J., Hérin R., Renard J., (1984), Géographie sociale. Ed. Masson, Paris, 388 p.

Gaillard J.C., (2003), «Territorial and ethno-cultural implications of a volcanic crisis: the case of the Mount Pinatubo eruption (Philippines) », in Alaya : Kapampangan Research Journal, 1/2003, pp.73-88.

Gill M-L., Pellegrin P., (2006), A Companion to Ancient Philosophy, chapitre 24, Ed. Wiley-Blackwell, Oxford, pp. 465485.

Godard O., Henry C., Lagadec P., Michel-Kerjan E., (2002), Traité des nouveaux risques. Précaution, crise, assurance, Paris, Ed. Gallimard, 217 p. 
Gosh A., (2005), «Entre douleur et révolte. Aux Andaman avec les oubliés du tsunami », Courrier international, publié le 26 janvier 2005 [URL : http://www.courrierinternational.com/article/2005/01/27/aux-andaman-avec-les-oublies-dutsunami], consulté le 11 septembre 2017.

Guéna P., (2017), «Vu du Moyen Âge : 1333, Florence inondée », The Conversation, mis en ligne le 03 septembre 2017, [URL : https://theconversation.com/vu-du-moyen-age-1333-florence-inondee83390?utm_medium=email\&utm_campaign=Newsletter du 4 septembre 2017 - 82216688\&utm_content=Newsletter $\mathrm{du}$ 4 septembre 2017

82216688+CID_fd70af43fd1756ab258efa252eadcc8d\&utm_source=campaign_monitor_fr\&utm_term=Vu du Moyen ge 1333 Florence inonde], consulté le 04 septembre 2017.

Guénard F., Simay P., (2011), « Du risque à la catastrophe. À propos d'un nouveau paradigme », La Vie des idées, mis en ligne le 23 mai 2011 [URL : http://www.laviedesidees.fr/Du-risque-a-la-catastrophe.html], consulté le 24 février 2017.

Gumuchian H., Marois C., Fevre V., (2000), Initiation à la recherche en géographie : aménagement, développement territorial, environnement, Montréal, Presses de l'Université de Montréal, 425 p.

Hans J., (1979), Le Principe responsabilité : une éthique pour la civilisation technologique, J. Greisch (trad.), Ed. du Cerf, 1990, pp. 13-30.

Hernandez J., (2008), «Le tourisme macabre à La Nouvelle-Orléans après Katrina : résilience et mémorialisation des espaces affectés par des catastrophes majeures », Norois, mars 2008, n²08, pp. 61-73, mis en ligne le 01 novembre 2010, [URL : http://norois.revues.org/2208], consulté le 11 septembre 2017.

Jousse G., (2009), Traité de riscologie - La science du risque, Ed. Imestra, 598 p.

Kervern G.Y., (1999), Eléments fondamentaux des cindyniques, Economica, 112 p.

Kervern G.Y., Boulanger P., (2007), Cindyniques, concept et mode d'emploi, Economica, 101 p.

Kervern G.Y., Rubise P., (1991), L'archipel du danger, Economica, 460 p.

Labbe T., (2017), Les Catastrophes naturelles au Moyen Age, Ed. CNRS, Coll. Histoire, 300 p.

Lacoste Y., (1982), «Terres à hauts risques », Herodote, n²4, Janvier-avril, p. 9.

Laquièze A., (2012), «L'introduction du principe de précaution dans la Constitution : sens ou non-sens ? », Revue de métaphysique et de morale, avril, ${ }^{\circ} 76$, pp. 549-562, [URL: http://www.cairn.info/revue-de-metaphysique-et-demorale-2012-4-page-549.htm], consulté le 10 septembre 2017.

Laurini R., Servigne S., (2011), «Leçons tirées d'une expérience de chorémisation automatique », SAGEO 2011 International Conference on Spatial Analysis and GEOmatics, Conférence internationale de Géomatique et d'Analyse Spatiale, Juillet 2011, Paris, pp.1-15 [https://hal.archives-ouvertes.fr/hal-01354482], consulté le 17 juin 2018.

Lecomte A., (2015), «L'inondabilité comme moteur de projet urbain », Actes des matinées du CGEDD, septembre 2015, La Défense, Paris, [URL: http://www.cgedd.developpementdurable.gouv.fr/IMG/pdf/compte_rendu_inondabilite_def_CR_cle78615d.pdf], consulté le 18 juin 2018.

Lefebvre G., (1932), La Grande Peur de 1789, Ed. Armand Colin, Paris, 272 p.

Leibniz, (1999) Essais de théodicée sur la bonté de Dieu, la liberté de l'homme et l'origine du mal. Publié en 1710, Ed. Flammarion, $506 \mathrm{p}$.

Leone F., Vinet F., (2011), «La vulnérabilité, un concept fondamental au cœur des méthodes d'évaluation des risques naturels », dans La vulnérabilité des sociétés et des territoires face aux menaces naturelles : Analyses géographiques, (dir.), Presses Universitaires de la Méditerranée, Coll. Géorisques, 144 p.

Létoublon F., (2012) «"Il meurt jeune, celui que les dieux aiment", Les héros sont des hommes comme les autres», Épopées du monde. Pour un panorama (presque) général, Feuillebois Pierunek E. (dir.), Classiques Garnier, coll. Rencontres, Paris, pp. 277-296.

Lévi-Strauss C., (1952), Race et histoire, chapitre 5, «L'idée de progrès », chapitre 10 : «Le double sens du progrès », Paris, UNESCO, 85 p.

Lévy J., (2000), « Revisiter le couple géographie physique-géographie humaine », dans Natures Sciences Sociétés, vol. 8, $\mathrm{n}^{\circ} 3$, juillet-septembre, pp. 78-79.

Liéval C., (2006), Les «nouveaux risques» en géographie. Entre évolutions et blocages, faut-il changer de paradigmes ?, mémoire de master 2, Grenoble, Institut de Géographie Alpine, juillet 2006, sous la direction de Soubeyran O., 89 p. 
Morin E., (2007), Ou va le monde ?, Paris, Ed. L'Herne, 108 p.

Morin E., (2007), Vers l'abîme, Paris, Ed. L’Herne, 181 p.

Morin E., (2009), «Edgar Morin : une année propédeutique de culture générale », dans Le Monde, le 12 mai 2009, [URL : http://internationalnews.over-blog.com/article-31357073.html], consulté le 12 septembre 2010.

Nora P., (1997), Les lieux de mémoire. Ed. Gallimard, Coll. Quarto, tome I, 1652 p.

November V., (2000), Les territoires du risque. Le risque comme objet de réflexion géographique, Bern, Peter Lang SA, $332 \mathrm{p}$.

Ozer P., De Longueville F., (2005), «Tsunami en Asie du Sud-Est : retour sur la gestion d'un cataclysme naturel apocalyptique », Cybergeo, «Environnement, Nature, Paysage », document 321, mis en ligne le 14 octobre 2005, [URL : http://cybergeo.revues.org/3081; DOI : 10.4000/cybergeo.3081], consulté le 27 août 2017.

Pascual A., Dagot, L., Vallée, B., \& Guéguen, N., (2009), « Soumission sans pression, médiatisation d'un tsunami et don d'argent: efficacité comparée de la porte-au-nez et du "vous êtes libre de..." », Revue Européenne de Psychologie Appliquée, $\quad \mathrm{n}^{\circ} 59, \quad \mathrm{pp}$ [URL: https://www.researchgate.net/publication/236784288_Soumission_sans_pression_mediatisation_d\%27un_tsunami_et_ don_d\%27argent_efficacite_comparee_de_la_porte-au-nez_et_du_vous_etes_libre_de], consulté le 27 août 2017

Pietralunga C., et Bonnefous B., (2017), «Mélenchon et Ciotti réclament une commission d'enquête sur la gestion

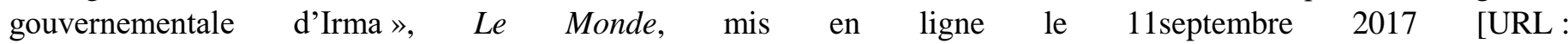
http://www.lemonde.fr/planete/article/2017/09/11/melenchon-et-ciotti-reclament-une-commission-d-enquete-sur-lagestion-gouvernementale-d-irma_5183742_3244.html\#vv4osHT6JXEeQj35.99], consulté le 13 septembre 2017.

Pigeon P., (2003a), «L'intérêt du risque pour l'enseignement en géographie », dans Les Risques, Moriniaux V. (dir.), Paris, Editions du temps, p. 9.

Pigeon P., (2003b), Géographie des Risques. Tome 1, Agrégations externes d'histoire et de géographie, Paris, CNED, $261 \mathrm{p}$.

Poirier J.P., (2005), Le tremblement de terre de Lisbonne., Ed. Odile Jacob, Paris, 284 p.

Reymond P., Margot A., Margot J., (2017), Les limites de l'aide humanitaire, mémoire de master, sous la dir. de Reinhard M., Ecole Polytechnique de Lausanne, Lausanne, 59 p.

Rochefort R. (1983), « Réflexions liminaires sur la géographie sociale », Actes du Colloque de Lyon (1983), Géographie Sociale, 14-16 octobre 1982, éd. Daniel Noin, Paris, pp. 11-15.

Roncayolo M., Bertran de Balanda S., (2016), Le géographe dans sa ville, Ed. Parenthèses, Coll. Architectures, Marseille, $256 \mathrm{p}$.

Roubineau J.-M., (2016), Milon de Crotone ou l'invention du sport, Ed. PUF, 392 p.

Sabourin C., (2010), «Avec le séisme, l'art haïtien a perdu une part de sa naïveté », La Presse.ca, publié le 25 avril 2010, [URL : http://www.lapresse.ca/international/dossiers/seisme-en-haiti/201004/25/01-4274049-avec-le-seisme-larthaitien-a-perdu-une-part-de-sa-naivete.php], consulté le 27 août 2017.

Verley P., (1997), La Rrévolution industrielle, Ed. Gallimard, Coll. Folio/Histoire, Paris, 1997, 544 p.

Veschambre V., (2006), «Penser l'espace comme dimension de la société. Pour une géographie sociale de plain-pied avec les sciences sociales », dans Séchet R., Veschambre V., Penser et faire la géographie sociale. Contribution à une épistémologie de la géographie sociale, Rennes, Presses Universitaires de Rennes, 397 p.

Veyret Y. (dir.), Beucher S., Reghezza M., (2004), Les risques. Comprendre, rechercher, s'entraîner, Ed. Bréal, Coll. Amphi Géographie, 206 p.

Veyret Y., Chocat B., (2005) «Les mégapoles face aux risques et aux catastrophes naturelles », La jaune et la rouge, dossier Environnement, «Les mégalopoles», juin-juillet 2005, n606, pp. 38-43 [URL: https://www.lajauneetlarouge.com/article/les-megapoles-face-aux-risques-et-aux-catastrophesnaturelles\#.Wy1hpadKh9M], consulté le 17 juin 2018.

Veyret Y., Reghezza M., (2005a), «Aléas et risques dans l'analyse géographique », Annales des Mines, série responsabilité et environnement, $\mathrm{n}^{\circ} 40$, octobre 2005, pp. 61-70.

Veyret Y., Reghezza M., (2005b), «L'émergence du risque en géographie », Cahiers Nantais, Risques et problématiques géographiques, $\mathrm{n}^{\circ} 64$, pp. 3-9.

Veyret-Mekdjian Y., (2001), « Géographie des risques naturels », La Documentation photographique, n8023, Paris, La Documentation française, octobre 2001, 63 p.

(C) 2018 ISTE OpenScience - Published by ISTE Ltd. London, UK - openscience.fr 